\title{
Is positron emission tomography for the management of potentially operable non-small cell lung cancer an efficient use of Quebec health care resources?
}

\author{
Andre Lamy MD FRCSC MHSc ${ }^{1,3}$, Amiram Gafni PhD²,3
}

$I^{n}$ $\mathrm{n}$ this issue of the Canadian Respiratory Journal, Nguyen et al (pages 19-25) evaluated the economic impact and costeffectiveness of positron emission tomography (PET) scanning in the management of potentially operable non-small cell lung cancer (NSCLC) in Quebec. Using a decision tree approach, they compared two strategies: chest computed tomography (CT) alone versus CT with whole-body PET. They found that CT with PET costs, on average, $\$ 1,268$ more than would CT alone, but would extend life expectancy by slightly more than three months $(0.27$ years $)$. The incremental cost-effectiveness ratio (ICER) was $\$ 4,689$ and the budget impact after taking the number of new cases of mediastinal metastases into account was $\$ 8,613,693$. Based on an acceptable threshold value of $\$ 50,000$ per life-year gained, the authors claim "our model shows that the use of PET to detect local and distant metastases in NSCLC is an intervention that would require an acceptable investment for each life year gained". In the present editorial, we review the economic question that decision-makers face and explain why the analysis used by Nguyen et al does not provide useful information for decision-makers.

Economics is based on three fundamental concepts: scarcity (whatever resources are available to health care, they are insufficient to support all possible activities), choice (because resources are scarce, we must choose between different ways of using them) and opportunity cost (by choosing to use resources in one way, we forgo other opportunities to use the same resources). On the basis of these concepts, resources are used efficiently if, and only if, the value of what is gained in using resources exceeds the value of what is forgone by not using them in all other ways. Thus, economic analysis should determine if "the value of what is gained from an activity outweighs the value of what has to be sacrificed" (1).

Scarcity, choice and opportunity cost reflect the nature of the problem faced by decision-makers with regard to questions such as those discussed by Nguyen et al. Cost-effectiveness analysis (CEA) has been presented in the research literature as a methodology to help decision-makers allocate a limited pool of resources. The underlying premise of CEA is that for a given level of resources available, society or the decision-maker wishes to maximize the total health benefit conferred (2-4). Hence, it seems to answer the decision-maker's question. The analytical tool of CEA is the ICER, which is calculated by dividing the difference in costs between the new and old treatments by the difference in effects, to yield the additional cost per unit of outcome (eg, \$4,689 per life-year saved from Nguyen et al). Two decision rules have been presented in the literature to determine whether a new program is worth implementing: the league table approach and the threshold approach. Under the league table approach, programs are adopted in a descending order of ICER until all available resources are exhausted. Under the threshold approach, if the program's ICER is lower than the threshold (eg, $\$ 50,000$ per life-year saved in Nguyen et al) it should be adopted.

However, the question facing the decision-maker is not simply a choice between CT alone versus CT with PET for NSCLC. A positive ICER, as resulted in Nguyen et al, means that resources used by the current intervention are not sufficient to cover the total costs of the new intervention for the same number of patients. The simple reality is that when overall funds are fixed, the additional funds required for a new program must come from other uses, ie, cuts to other programs. The use of ICER ignores that simple reality, because the total costs and effects of all the programs involved, including those that will have to be cut, are not included in the calculations or the decision rule. Instead, a value judgement is made, either implicitly or explicitly, about whether an ICER represents a 'good buy' (ie, falls under a threshold), as was the case in Nguyen et al. But funding new interventions that have 'acceptable' ICERs requires, and hence leads to, continuous increases in program expenditures (eg, over $\$ 8$ million as in Nguyen et al), because the new, more costly technology is added without other programs being cut to generate sufficient resources for the new program (5).

Economics provide valid methods for maximizing the health improvements that can be attained with a given allocation of resources by taking into account the opportunity cost of these resources $(6,7)$. These methods can help decision-makers allocate health resources efficiently under circumstances of fixed, shrinking or increasing budgets. Although the data requirements for these methods may be substantial, they reflect the complexity of the question being addressed. A less 'data hungry' approach is available for use in practical decisionmaking (6-8). According to this approach, to determine if an intervention is worth pursuing, one has to know what will be the total additional costs and effects to the system as a result of implementing a new, more costly, but more effective, intervention. Also, one must identify an existing intervention (or

\footnotetext{
${ }^{1}$ Department of Surgery; ${ }^{2}$ Centre for Health Economics and Policy Analysis, ${ }^{3}$ Department of Clinical Epidemiology and Biostatistics, McMaster University, Hamilton, Ontario

Correspondence and reprints: Dr Andre Lamy, General Site, McMaster Clinic Room 704, McMaster University, 237 Barton Street East,

Hamilton, Ontario L8L 2X2. Telephone 905-522-0175, fax 905-522-2029, e-mail lamyand@hhsc.ca
} 
combination of interventions) that, if cancelled, will generate the additional resources necessary to implement the new intervention and will reduce the community health-related wellbeing by less than the incremental gain produced by the new intervention.

Nguyen et al estimated that the additional annual budgetary burden for Quebec would be over $\$ 8$ million. However, they did not identify where this money would come from. In other words, to claim that the use of CT with PET for patients with NSCLC represents an efficient use of Quebec's health care resources, they should have identified an intervention (or set of interventions) that, when cancelled, would free up enough resources to implement the new intervention (ie, free up $\$ 8.6$ million every year) and reduce the community health-related well-being by less than the incremental gain produced by CT with PET for NSCLC. Without doing so, they failed to address the decisionmaker's problem.

\section{REFERENCES}

1. Williams A. The economic role of health indicators. In: Teeling Smith G, ed. Measuring the Social Benefit of Medicine. London: Office of Health Economics, 1983:63-7.

2. Weinstein MC, Stason WB. Foundation of cost-effectiveness analysis for health and medical practices. N Engl J Med 1977;296:716-21.

3. Gold MR, Siegal JE, Russel LB, Weinstein MC. Cost Effectiveness in Health and Medicine. New York: Oxford University Press, 1996.

4. Williams A. What Could Be Nicer Than NICE? London: The Office of Health Economics, 2004.

5. Gafni A, Birch S. Inclusion of drugs in provincial drug benefit programs: Should "reasonable decisions" lead to uncontrolled growth in expenditures? CMAJ 2003;168:849-51.
It has been argued that decision makers "should maintain a healthy skepticism about the results of cost-effectiveness analyses and the usefulness of those results in purchasing and planning decisions" (9). In our opinion, this problem arises because often the (economic) analysis performed is based on a noneconomical way of interpreting economic information, as explained previously. As we have shown elsewhere, the use of such methods have resulted in Canada (5) and other countries (10) having uncontrolled growth in expenditures without evidence of any increase in total health improvements; increased inequalities in the availability of services; and concerns about the sustainability of public funding for new technologies. As Williams nicely observed (4), “...reality is horrendously complicated...the more complex the reality is, the more dangerous it is to rely on short cuts rather than careful analysis". The use of simple tools, such as the ICER, represents a departure from the economic discipline, fails to address the decision-maker's problems and is harmful.

6. Birch S, Gafni A. Cost effectiveness/utility analysis: Do current decision rules lead us to where we want to be? J Health Econ 1992;11:279-96.

7. Sendi P, Gafni A, Birch S. Opportunity cost and uncertainty in the economic evaluation of health care interventions. Health Econ 2002;11:23-31.

8. Gafni A, Birch S. Guidelines for the adoption of new technologies: A prescription for uncontrolled growth in expenditures and how to avoid the problem. CMAJ 1993;148:913-7.

9. Naylor D. Cost-effectiveness analysis: Are the outputs worth the inputs? ACP J Club 1996;124:A12-4.

10. Gafni A, Birch S; NICE; National Health Service. NICE methodological guidelines and decision making in the National Health Service in England and Wales. Pharmacoeconomics 2003;21:149-57. 


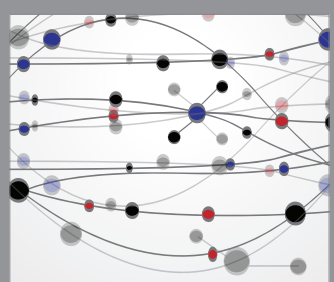

The Scientific World Journal
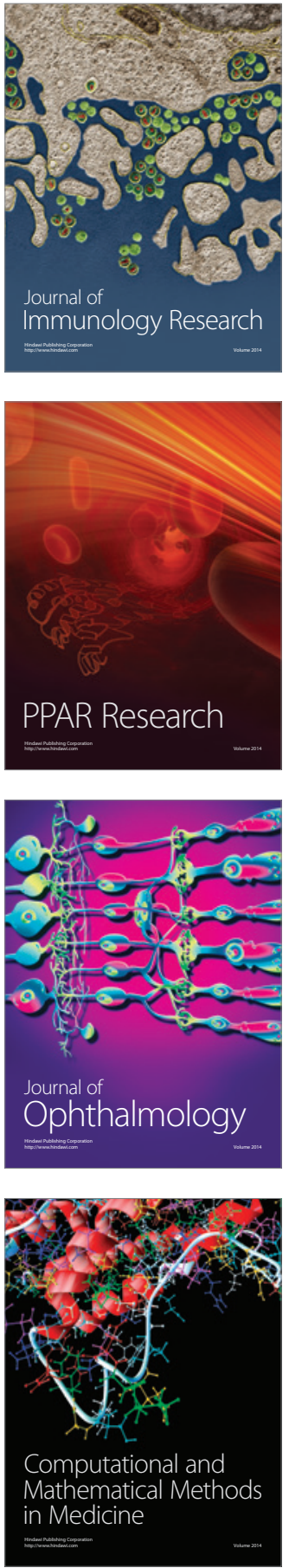

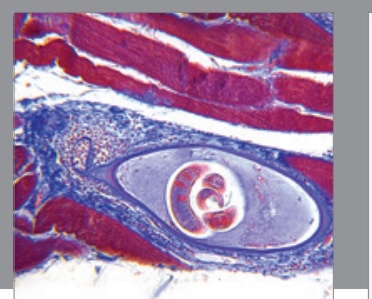

Gastroenterology Research and Practice

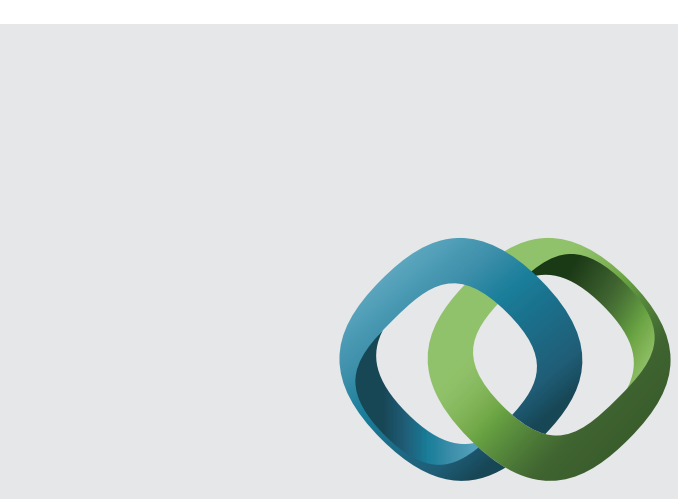

\section{Hindawi}

Submit your manuscripts at

http://www.hindawi.com
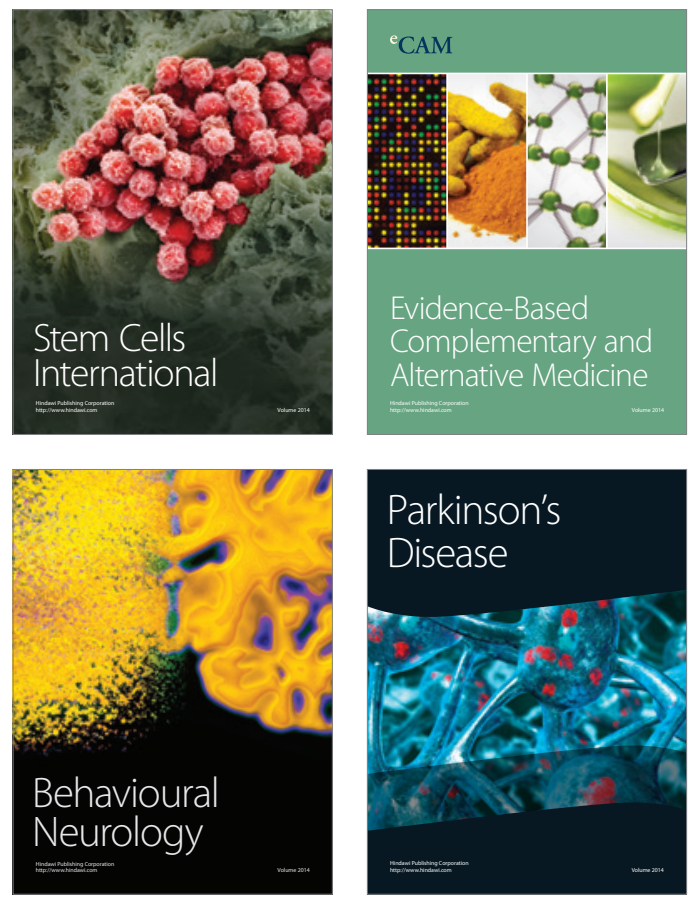
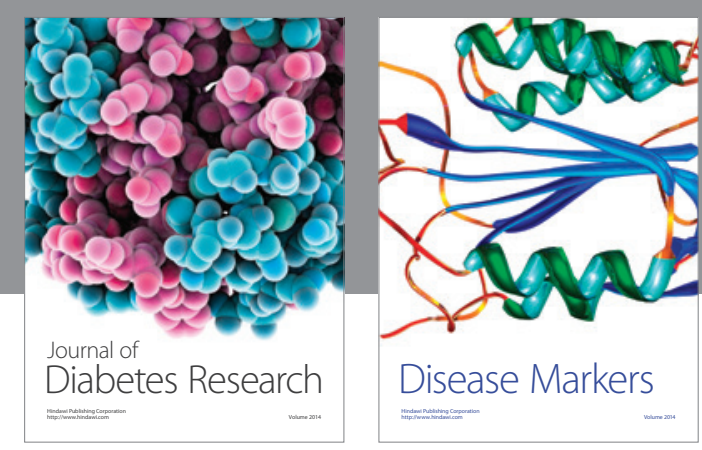

Disease Markers
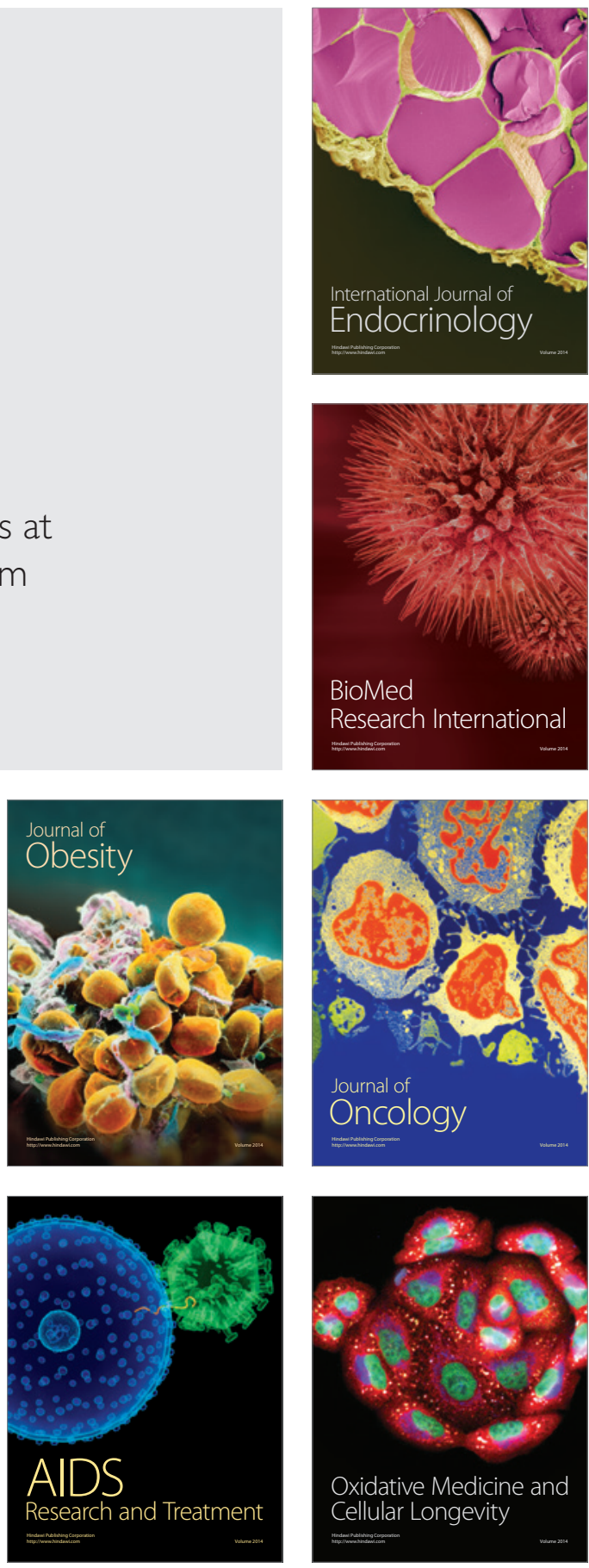